\title{
LITERATUR
}

\section{Interdisziplinäre Betrachtungen europäischer und internationaler Weltraumpolitik}

\author{
Markus Hesse*
}

Weltraumpolitik ist in erster Linie eine Angelegenheit von Nationalstaaten. Die Rolle von internationalen Gremien und Institutionen ist ihnen gegenüber in den Internationalen Beziehungen nachgeordnet, solange internationales Weltraumrecht nicht stark ausgeprägt ist und kaum weiterentwickelt wird. Vor diesem Hintergrund sind der Aufstieg neuer Weltraummächte, unter ihnen ,Europa', verstanden als eine Kooperation von Europäischer Union und Europäischer Weltraumagentur (ESA), sowie die sich daraus ergebenden neuen Machtkonstellationen besonders interessant. Es ist in erster Linie das einzigartige Governance-Modell der Zusammenarbeit zwischen Europäischer Union und ESA, das eine Vorbildfunktion für andere nicht-staatliche Ebenen von Weltraumpolitik haben kann und deshalb eine besondere Aufmerksamkeit verdient.

Der Weltraumrat, bestehend aus Vertretern der Europäischen Union und der ESA auf Ministerebene, hat 2007 die erste europäische Resolution zur Weltraumpolitik verabschiedet. ${ }^{1}$ Dieses Dokument ist ein bedeutender Meilenstein, vor allem auf dem Weg zu einer gemeinsamen europäischen Weltraumpolitik, aber auch zur Konstruktion einer europäischen Identität mittels der Weltraum- und Raumfahrtpolitik von Europäischer Union und ESA. Seit dem Inkrafttreten des Vertrags von Lissabon 2009 ist die Europäische Union gemäß Art. 189 AEUV verpflichtet, mit der ESA in Weltraumangelegenheiten zu kooperieren. In der Europäischen Union ist Weltraumpolitik
Christophe Venet/Blandina Baranes (Hrsg.): European Identity through Space. Space Activities and Programmes as a Tool to Reinvigorate the European Identity. Studies in Space Policy, Band 9, herausgegeben vom European Space Policy Institute (ESPI), Springer: Wien/New York 2012, ISBN 978-3-7091-0975-5, 340 Seiten, 128,35 €.

Bertrand de Montluc: A New International Strategic Context for Space Policies, Nova Science Publishers: New York 2011, ISBN 978-1-61122-081-0 (eBook), 99 Seiten, 61,99 €.

nunmehr eine geteilte Zuständigkeit, die auf dieser Grundlage neben den nationalen Weltraumpolitiken parallel ausgeführt wird. Mit diesem klaren politischen Auftrag arbeiten Rat, Parlament und Kommission - wenn auch nicht immer einvernehmlich und mit den gleichen Zielsetzungen - an einer Weiterentwicklung der europäischen Weltraumpolitik. Diese Ausgestaltung und Ausweitung der europäischen Weltraumpolitik steht immer in Wechselwirkungen zu den Politiken der etablierten Weltraummächte, allen voran der USA, Russland und China, sowie den anderen aufstrebenden Staaten mit neuen Weltraumprogrammen und vergleichsweise jungen Weltraumagenturen, wie Indien, Japan, Brasilien oder Nigeria.

Vor diesem Hintergrund untermauerte die Europäische Kommission 2011 die europäischen Ambitionen auf der weltraumpolitischen Bühne in ihrer Mitteilung „Auf dem Weg zu einer Weltraumstrategie der Europäischen Union im

* Dr. Markus Hesse, Projektmanager, Stiftung Mercator, Essen.

1 Rat der Europäischen Union: Beratungsergebnisse der Tagung des Rates (Wettbewerbsfähigkeit) vom 21.22. Mai 2007 - Entschließung zur Europäischen Raumfahrtpolitik, Dok. 10037/07. 
Dienst der Bürgerinnen und Bürger", 2 in der die herausragende Bedeutung der Weltraumpolitik betont und die Erarbeitung eines gemeinsamen europäischen Weltraumprogramms gefordert wird. Bei dieser Weiterentwicklung werden die europäischen Bürger aber nicht abgekoppelt, wenn man die Ergebnisse des repräsentativen Eurobarometer 2012 zu „Space Activities“ betrachtet. ${ }^{3}$ Demnach sind zumindest einem Teil der EU-Bürger die Bedeutung und die positiven Eigenschaften von zahlreichen Weltraumanwendungen, beispielsweise in den Bereichen Navigation, Erdbeobachtung und Kommunikation, bewusst.

\section{Der Beitrag von Weltraumpolitik zu einer europäischen Identität}

In diesen Zeitraum fällt auch der Sammelband „European Identity through Space" 4 aus dem Jahr 2012, der im Nachklang einer Konferenz zum gleichen Thema am renommierten European Space Policy Institute (ESPI) in Wien im November 2009 entstand. Die Konferenz und dieser Sammelband sind vor allem interessant, da sie erstmals die gegenseitigen Wechselwirkungen zweier großer Themenbereiche - europäische Weltraumpolitik einerseits und europäische Identität andererseits - betrachten. Diese Schnittmengen zu untersuchen hat einen besonderen Reiz, da weder der Aufbau und die Organisation der europäischen Weltraumpolitik noch die Konstruktion einer europäischen Identität ansatzweise abgeschlossen sind. Bereits jetzt die Auseinandersetzung mit diesen Themen zu suchen, ist dennoch eine bedeutende Herausforderung für die Forschung und ein erstes Verdienst der Herausgeber sowie der beteiligten Autoren.

Die Interdependenz von Weltraumpolitik und Identität ist das zentrale Thema dieses Sammelbandes. Somit beschäftigen sich nahezu alle Autoren mit den übergreifenden Ausgangsfragen: Wie kann Weltraumpolitik einen Beitrag zur Identitätsbildung in Europa leisten? Welche (Rück-)Wirkungen hat eine sich herausbildende gemeinsame europäische Identität auf die europäische Weltraumpolitik? Dabei haben die Autoren die Schnittmengen von Weltraumpolitik und Identität in der Regel vor ihrem eigenen disziplinären Hintergrund heraus beleuchtet. Im ersten Abschnitt des Buches finden sich zehn Autoren mit einem fachlichen Hintergrund zur Weltraumpolitik, im zweiten Abschnitt fünf Autoren, die vorwiegend den Sozialwissenschaften zuzuordnen sind. So beleuchtet Werner Balogh im ersten Abschnitt „Europe as an actor in the United Nations Committee on the Peaceful Uses of Outer Space"5 (UNCOPUOS) und stellt dabei überzeugend die aufstrebende Rolle und die wichtigen Beiträge der europäischen Mitgliedstaaten in diesem Gremium der Vereinten Nationen dar. Ebenfalls im ersten Abschnitt behandeln Stephan Lingner im Artikel „European identity, technology and society. The case for space“6 und Mathias Spude in „Does the European integrated space industry contribute to a new European identity?"7 Fragen zu Weltraumtechnologie und -industrie. Beide stellen die Potenziale von Hochtechnologie heraus, wenn es um die Konstruktion einer europäischen oder in letzter Konsequenz sogar welt-

2 Europäische Kommission: Mitteilung der Kommission an das Europäische Parlament, den Rat, den Europäischen Wirtschafts- und Sozialausschuss und den Ausschuss der Regionen. Auf dem Weg zu einer Weltraumstrategie der Europäischen Union im Dienst der Bürgerinnen und Bürger, KOM (2011) 152.

3 Europäische Kommission: Flash Eurobarometer 355. Space Activities. Report, Fieldwork: July 2012, September 2012.

4 Christophe Venet/Blandina Baranes (Hrsg.): European Identity through Space. Space Activities and Programmes as a Tool to Reinvigorate the European Identity. Studies in Space Policy, Band 9, herausgegeben vom European Space Policy Institute (ESPI), Wien/New York 2012.

5 Werner Balogh: Europe as an actor in the United Nations Committee on the Peaceful Uses of Outer Space, in: Christophe/Baranes (Hrsg.): European Identity through Space, 2012, S. 60-79.

6 Stephan Lingner: European identity, technology and society. The case for space, in: Christophe/Baranes (Hrsg.): European Identity through Space, 2012, S. 93-102.

7 Mathias Sprude: Does the European integrated space industry contribute to a new European identity?, in: Christophe/Baranes (Hrsg.): European Identity through Space, 2012, S. 102-118. 
weiten Identität geht. Aus dem zweiten stärker sozialwissenschaftlichen Abschnitt des Sammelbandes sei auf den Artikel „European identity through space - how to make public opinion instrumental ${ }^{168}$ von Marcus Hornung hingewiesen. Der Autor arbeitet die Interdependenz zwischen europäischer Weltraumpolitik und Identität heraus und belegt deren schwache Ausprägung und Perzeption bei der Bevölkerung Europas. Er schließt mit überzeugenden Empfehlungen, um diese Situation zu verbessern: Die Wahrnehmung von positiven Eigenschaften der Weltraumanwendungen sollte der Bevölkerung stärker vermittelt werden, beispielsweise durch Aufnahme des Themenfeldes in die Schulbildung, aber auch mittels einer transparenteren Kommunikation seitens der europäischen Institutionen.

Diese Aufteilung und interdisziplinäre Betrachtungsweise des Untersuchungsgebiets in dem vorliegenden Sammelband ist Fluch und Segen für den Leser zugleich. Einerseits werden so größere Teile der potenziell interessierten ,scientific community ' abgedeckt, die sich mit dem Themenbereich beschäftigen. Der Sammelband liefert also einen Werkzeugkasten mit zahlreichen und deutlich unterschiedlichen Methoden und Instrumenten für eine Annäherung an europäische Weltraumpolitik und Identität. Andererseits existiert kein gemeinsamer theoretischer oder konzeptioneller Rahmen der Autorengemeinschaft. Die zentralen Begriffe, allen voran ist das in diesem Sammelband „Identität", werden - trotz des hilfreichen Abschnitts „European identity: attempt of a definition"9 - immer wieder unterschiedlich interpretiert und in den Artikeln anders verwendet, sodass dem Leser teilweise der Zugang zu fachfremden Abschnitten erschwert wird. Andererseits ist diese Begriffsvielfalt ein Anreiz für den weltraumpolitisch schon, vorbelasteten' Leser, in diesem Band originär neue Blickwinkel und Argumentationen kennenzulernen.

Letztlich soll und kann der Sammelband auch einen Beitrag zum besseren Verständnis und zu größerer Anerkennung der verschiedenen Wissenschaftsdisziplinen untereinander leisten. Mit dem breiten interdisziplinären Ansatz, der über die ansonsten meist politik- oder ingenieurwissenschaftlich dominierten Sichtweisen hinausgeht, hat „European Identity through Space" das Potenzial, verschiedene wissenschaftliche und gesellschaftliche Gruppen zu erreichen. Neben diesem Band existiert derzeit keine wissenschaftliche Publikation, die diesen Ansprüchen im gleichen Maß gerecht wird und einen vergleichbaren Ansatz zugrunde legt.

\section{Die großen Weltraummächte in der internationalen Weltraumpolitik}

Der zweite hier zu besprechende Band zur internationalen Weltraumpolitik von Bertrand de Montluc trägt den Titel „A New International Context for Space Policies"10 und dürfte vor allem für die Schnittstellen des Fachbereichs Internationale Beziehungen zur Weltraumpolitik interessant sein. Das Buch ist in sechs inhaltliche Kapitel eingeteilt, deren Struktur sofort einleuchtet. Im ersten Kapitel werden die neuen technischen Möglichkeiten der Weltraumanwendungen in Bezug zu den Internationalen Beziehungen gesetzt. Anschließend wird der neue strategische Kontext der Weltraumpolitik im folgenden Kapitel aufgearbeitet und vorgestellt. In Kapitel drei bis sechs werden die größten Weltraummächte analysiert, also China, Russland, USA und Europa (wiederum verstanden als ESA und Europäische Union).

Der Autor legt zunächst die Auswirkungen des im Vergleich zum 20. Jahrhundert beschleunigten technologischen Fortschritts und der

8 Marcus Hornung: European identity through space - how to make public opinion instrumental, in: Christophe/ Baranes (Hrsg.): European Identity through Space, 2012, S. 215-227.

9 Blandina Baranes: European identity: attempt of a definition, in: Christophe/Baranes (Hrsg.): European Identity through Space, 2012, S. 10-24.

10 Bertrand de Montluc: A New International Strategic Context for Space Policies, Hauppauge 2011. 
Globalisierung auf die Weltraumpolitik überzeugend dar. Dabei bleibt er jedoch nicht eindimensional, sondern wägt gut nachvollziehbar zwischen den positiven Eigenschaften von Weltraumanwendungen und deren potenziell negativen Auswirkungen ab. Vor dem Hintergrund dieser Entwicklungen beobachtet de Montluc das verstärkte Aufkommen der im Vergleich zu den USA und Russland neuen Weltraummächte, allen voran China. Doch auch weniger einflussreiche Staaten wie Nigeria, Algerien, Argentinien, Malaysia oder Indonesien haben mittlerweile oder entwickeln derzeit eigene Weltraumprogramme. All dies wird in den nächsten Jahrzenten Auswirkungen auf die geostrategische Lage im Weltraum haben.

Die internationale Weltraumpolitik untersucht der Autor in den folgenden Kapiteln anhand der vier großen Weltraummächte. Dass China als erste in der Reihe steht, ist dabei kein Zufall. Vielmehr stellt de Montluc überzeugend dar, dass Weltraumprogramme in Asien, namentlich neben dem chinesischen sind das indische und das japanische gemeint, einen Bedeutungszuwachs erfahren. Dieser beinhaltet eine große Bandbreite an Projekten: bemannte Raumfahrt, naturwissenschaftlich-technische Experimente, zivil-militärische Anwendungen wie Telekommunikation oder Erdbeobachtung bis hin zu sicherheitspolitischen und militärischen Weltraumnutzungen. In Ergänzung eines sehr guten Überblicks über das chinesische Weltraumprogramm thematisiert der Autor hier auch verschiedene Blickwinkel, beispielsweise die Einschätzungen der anderen großen Weltraummächte zur chinesischen Weltraumpolitik.

Demgegenüber stellt sich die Situation in Russland anders dar. Als eine der alten Weltraummächte hat es besonders in den 1990er Jahren einen starken Bedeutungsverlust erfahren. Zahlreiche russische Weltraumprojekte wurden in dieser Zeit eingestellt oder zumindest reduziert. Dennoch skizziert de Montluc in dieser Hinsicht eine Wende in der zweiten Dekade des 21. Jahrhunderts: Russische Trä- gerraketen sind weltweit führend und in den Feldern Erdbeobachtung sowie Telekommunikation sieht der Autor großes Potenzial in den nächsten Jahren. Daraus resultiert auch die Annahme, dass Russland in den kommenden Jahren ein potenter und potenzieller Partner für die westlichen Weltraummächte, vor allem für den ehemaligen, größten Rivalen USA, werden könnte.

Die führende Weltraummacht, die im folgenden Kapitel vergleichsweise kurz ,abgearbeitet" wird, ist - trotz der grundsätzlichen Devise seit den 1960er Jahren ,America first! ' - einer internationalen Kooperation gegenüber nicht abgeneigt. Die Schwerpunkte der Obama-Administration liegen aber gemäß de Montlucs Analyse im Gegensatz zu früheren Zeiten nicht bei Weltraumwaffen, sondern eher bei Unterstützung der Klimaforschung und Weltraumaufklärung und unterscheiden sich vor allem in diesen Punkten gerade von der Vorgängerregierung.

Abschließend nimmt sich der Autor ,Europa als Weltraummacht vor, wobei die Differenzierung zwischen Programmen der ESA, der Europäischen Union oder nur einiger europäischer Nationalstaaten im Verbund teilweise schwierig erscheint. Dennoch hebt de Montluc mehrfach die Vorbildrolle Europas hervor, die vor allem die Governance der europäischen Programme wie Galileo oder Global Monitoring for Environment and Security (GMES) betrifft. Diese könnten einen Modellcharakter haben - nicht nur für zukünftige europäische Programme, sondern auch für Kooperationen mit den anderen Weltraummächten. Rein militärische Nutzungen von Weltraumanwendungen werden jedoch für die Weltraummacht Europa keine besondere Rolle spielen, da die ESA zwar die technologischen Voraussetzungen mitbringt, aber kein sicherheitspolitischer Akteur im internationalen System ist. Eine Einbindung von vereinzelten zivil-militärischen Programmen in die Gemeinsame Sicherheitsund Verteidigungspolitik (GSVP) der Europäischen Union ist dagegen vorstellbar. 
Auffallend ist die stark nationale Sichtweise des Autors auf die internationale Weltraumpolitik. So wird beispielsweise das UNCOPUOS nur selten erwähnt, weitere internationale Institutionen und Gremien oder beispielsweise weltraumrechtliche Ansätze zur Verständigung werden kaum berücksichtigt. De Montlucs Monografie bietet dem Leser dennoch einen sehr guten Überblick über die nationalen Weltraumprogramme der großen Weltraummächte. Zudem werden die verschiedenen Weltraumprogramme nicht nur beschrieben und darüber hinaus erläutert, sondern grundsätzlich in Relation zu den jeweils anderen na- tionalen Programmen und den Sichtweisen der anderen Weltraummächte gesetzt. Dies ist von besonderem Wert für eine weitere Auseinandersetzung mit den gegenseitigen Wechselwirkungen in der internationalen Weltraumpolitik und für weiterführende Analysen in den Internationalen Beziehungen. Die Monografie liefert eine kenntnisreiche Einführung in das Themenfeld und ist somit für eine intensive Beschäftigung und Auseinandersetzung mit der internationalen Weltraumpolitik - beispielsweise in der universitären Lehre - absolut zu empfehlen.

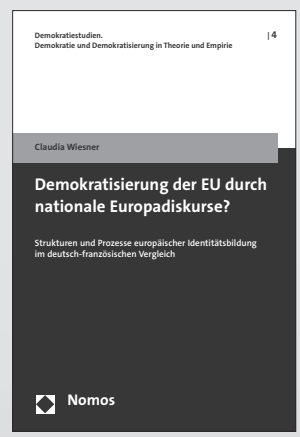

\section{Demokratisierung der EU durch nationale Europadiskurse?}

Strukturen und Prozesse europäischer Identitätsbildung im deutsch-französischen Vergleich Von Claudia Wiesner

2013, ca. 500 S., brosch., ca. 90,-€ ISBN 978-3-8487-0536-8

(Demokratiestudien. Demokratie und Demokratisierung in Theorie und Empirie, Bd. 4) Erscheint ca. November 2013

Können nationale Europadiskurse europäische Identität erzeugen und damit zur Demokratisierung der EU beitragen? Aufbauend auf einer ausführlichen vergleichenden Untersuchung nationaler Europadiskurse sowie der politischen Systeme und Kulturen Deutschlands und Frankreichs liefert der Band zahlreiche Ergebnisse sowie weiterführende Hypothesen und Modelle zu dieser Frage.

Bestellen Sie jetzt telefonisch unter 07221/2104-37. Portofreie Buch-Bestellungen unter www.nomos-shop.de/21043

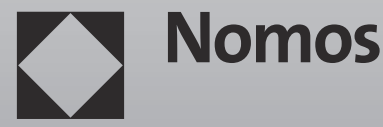

